\title{
Endovascular management of acute aortic dissection
}

\author{
Mamdouh Khayat ${ }^{1}$, Kyle J. Cooper ${ }^{1}$, Minhaj S. Khaja ${ }^{1}$, Ripal Gandhi ${ }^{2}$, Yolanda C. Bryce ${ }^{3}$, David M. \\ Williams ${ }^{1}$
}

${ }^{1}$ Department of Radiology, Division of Vascular and Interventional Radiology, University of Michigan Health Systems, Ann Arbor, MI, USA; ${ }^{2}$ Department of Radiology, Division of Vascular and Interventional Radiology, Miami Cardiac and Vascular Institute, Miami, FL, USA; ${ }^{3}$ Department of Radiology, Division of Vascular and Interventional Radiology, Memorial Sloan Kettering Cancer Center, New York, NY, USA

Contributions: (I) Conception and design: KJ Cooper, M Khayat; (II) Administrative support: None; (III) Provision of study material or patients: KJ Cooper, MS Khaja; (IV) Collection and assembly of data: KJ Cooper, M Khayat, MS Khaja; (V) Data analysis and interpretation: None; (VI) Manuscript writing: All authors; (VII) Final approval of manuscript: All authors.

Correspondence to: Kyle J. Cooper, MD. Department of Radiology, Division of Vascular and Interventional Radiology, University of Michigan Health Systems, Ann Arbor, USA. Email: kycooper@med.umich.edu.

\begin{abstract}
Acute dissection of the thoracic aorta is a potentially life-threatening condition which requires collaborative treatment from multiple specialties for optimal patient outcomes. Dissections involving the ascending aorta and aortic arch have traditionally been managed entirely by surgery, while dissections beyond the arch vessels have most commonly been relegated to medical management. This algorithm has been undergoing a paradigm shift over the past two decades due to improvements in stent graft technology, better understanding of the hemodynamic interactions of the true and false lumen and their influence on organ and limb perfusion, and improvements in medical management and long term surveillance for dissection-related complications. This manuscript includes a brief discussion of the pathogenesis and etiology of dissection, followed by an in-depth review of the medical and endovascular techniques utilized to treat patients afflicted by this condition.
\end{abstract}

Keywords: Aorta; dissection; fenestration; stent graft; malperfusion

Submitted Aug 28, 2017. Accepted for publication Sep 26, 2017.

doi: $10.21037 / \mathrm{cdt} .2017 .10 .07$

View this article at: http://dx.doi.org/10.21037/cdt.2017.10.07

\section{Introduction}

Considered a component of the "acute aortic syndrome" spectrum, aortic dissection is characterized by propagation of a hematoma within the aortic media. Though the precise pathophysiology is not conclusively reconciled, it is theorized that the hematoma arises spontaneously in the media and then propagates through the overlying intima to create an intimal tear, or the tear arises spontaneously or traumatically through the intima and introduces aortic blood into the intramedial space. If the intramedial space has both entry and exit tears, then the newly formed false lumen channel can conduct blood flow, and a membranelike dissection flap can be observed by cross-sectional imaging. This results in the characteristic pathology of aortic dissection. If the hemorrhage remains thrombosed, the process is called intramural hematoma (IMH). The intramural hemorrhage, whether classical dissection or $\mathrm{IMH}$, weakens the structural integrity of the aortic wall, predisposing it to both false lumen aneurysmal degeneration and rupture. Acute aortic rupture represents the most common cause of death in patients with aortic dissection (1).

Although the etiology of aortic dissection is multifactorial, hypertension is considered the most prevalent risk factor (2). Other associated risk factors include smoking history, dyslipidemia, deceleration trauma, certain drugs (i.e., cocaine), and connective tissue disorders $(3,4)$. The incidence of aortic dissection is $3-4$ cases per 100,000 patients per year according to the International Registry of Acute Aortic Dissections (IRAD), with a welldocumented male predilection (5-7). 

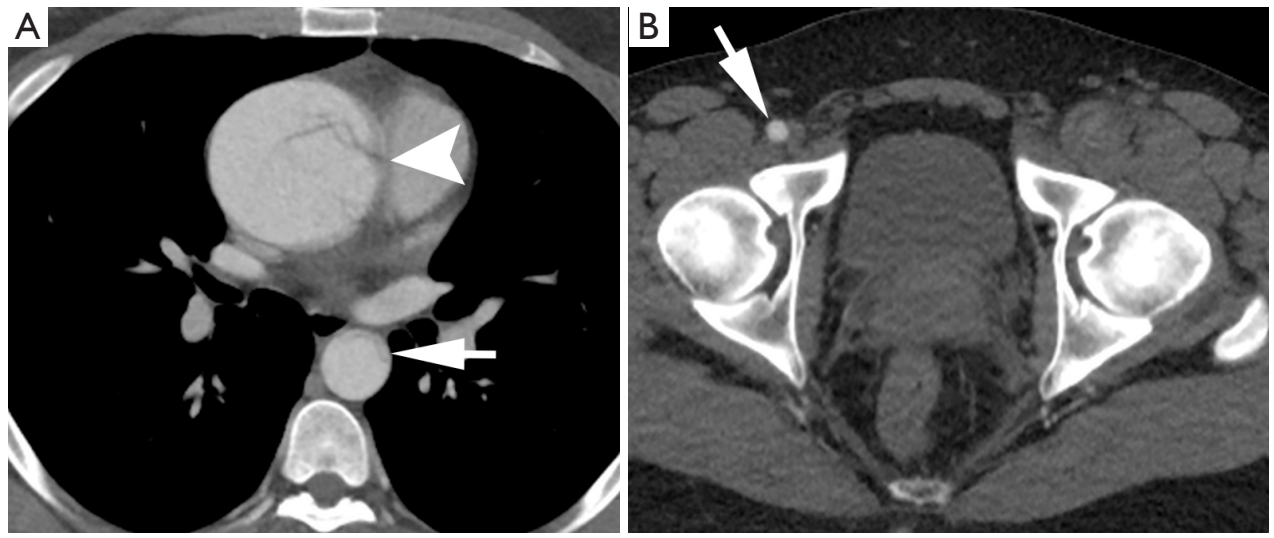

Figure 1 Contrast-enhanced CT angiography (CTA) of a 29-year-old man with history of Marfan syndrome and hypertension, presenting with severe chest pain radiating to his abdomen with transient paresthesia of left lower extremity. (A) Thoracic imaging confirms an aneurysmal type A aortic dissection originating at the aortic root (arrowhead) and true lumen collapse in descending thoracic aorta (arrow); (B) at the level of the pelvis, note is made of a patent right common femoral artery (arrow) and an occluded left common femoral artery.

\section{Diagnosis}

Patients typically present with acute-onset severe chest or back pain, often described as "tearing," that can be migratory. Depending on the location of the dissection, additional diagnostic cues may include neurologic deficits, visceral pain or dysfunction from malperfusion, pulse differentials, and a murmur from aortic valve insufficiency.

Diagnostic imaging plays a pivotal role in confirmation of diagnosis and the classification and localization of intimal tears. Imaging can help direct care and gauge level of urgency, particularly if there is pericardial, mediastinal, or pleural hemorrhage. Contrast-enhanced computed tomography angiography (CTA) is often the imaging modality of choice, as it is readily available, non-invasive, reproducible, and prompt (8). Moreover, CTA has superior spatial resolution for the characterization of dissection flaps and evaluation of luminal patency (Figure 1). Other diagnostic options include echocardiography (transthoracic or transesophageal) and magnetic resonance angiography (MRA). Nonetheless, acuity of presentation and gravity of clinical picture dictates course of care and necessity of preoperative imaging. In more acute critical presentations, invasive angiography can function as the initial diagnostic imaging modality prior to further intervention.

\section{Classification}

Classification of aortic dissection is based on anatomical location of the affected aorta. The Stanford Classification categorizes dissections based on involvement of the ascending aorta or arch (type A), defined as the aorta proximal to the lateral border of the left subclavian, or isolated to the descending thoracic aorta (type B). There can be further sub-classification based on chronicity. Acute dissections are defined as those with less than 14 days since onset of symptoms; dissections are considered chronic if symptoms have been present longer than 2 weeks, a temporal distinction from the era of open surgical repair needing clarification and perhaps revision in the era of endovascular treatment. Moreover, dissections can be categorized as complicated or uncomplicated; complicated dissections include those with rupture or impending rupture, severe aortic valve insufficiency, coronary or limb or visceral malperfusion, unmanageable hypertension, cardiac tamponade, neurological deficits, or refractory pain.

Of important distinction in aortic dissection complicated by malperfusion is delineation of the pathophysiologic mechanism of branch-vessel compromise. This can be categorized as static or dynamic obstruction (Figure 2). In static obstruction, the dissection intersects and enters the branch vessel with propagation of the false lumen and subsequent constriction of the true lumen. Alternatively, in dynamic obstruction, the plane of dissection spares the branch origin, with the dissection flap prolapsing over the branch ostium and impeding flow to the branch vessel (9). This distinction is important as it dictates endovascular treatment options.

Type A dissections are associated with a higher mortality of approximately $1 \%$ to $2 \%$ per hour following symptom 


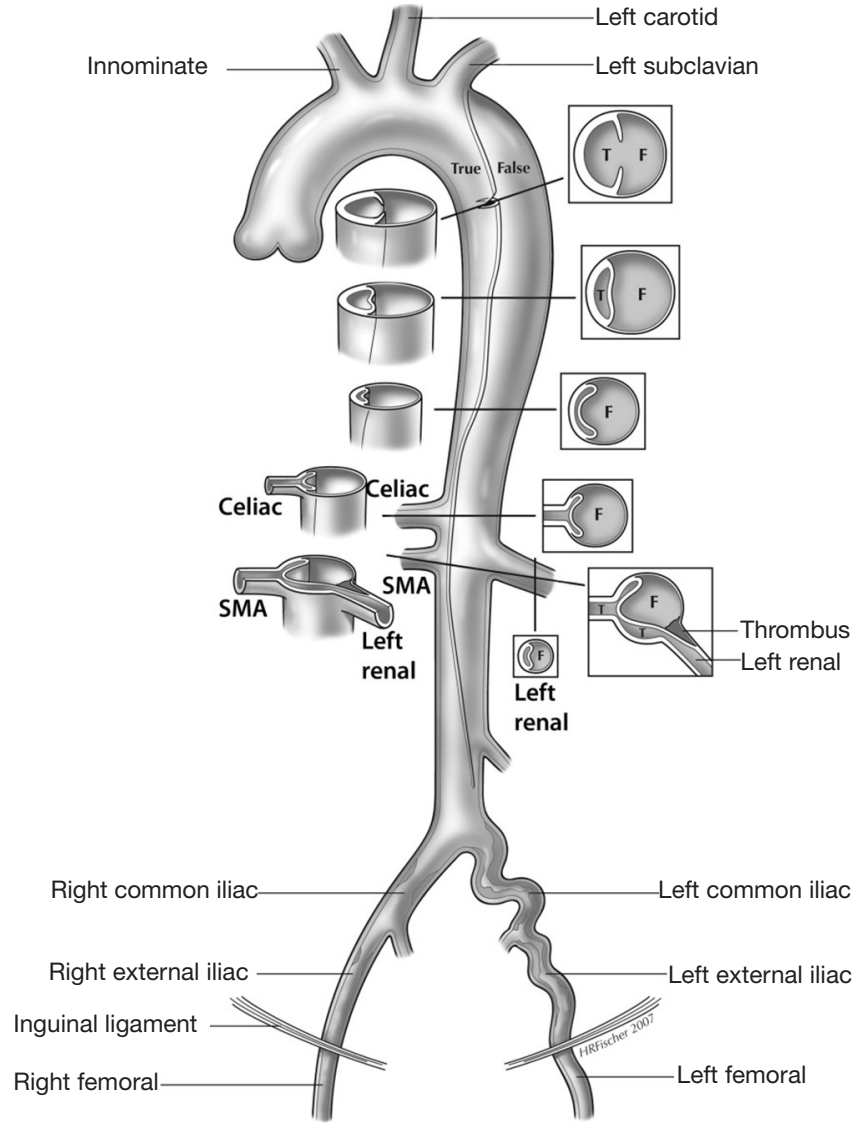

Figure 2 A schematic diagram of type B aortic dissection is depicted. The primary entry tear is localized to the proximal descending thoracic aorta. The dissection flap distal to this shows evidence of collapse of the true lumen, resulting in dynamic obstruction of the celiac and superior mesenteric arteries. In dynamic obstruction, the dissection does not extend into the branch vessels but rather occludes them by intermittent obstruction of the flap during the cardiac cycle. In contrast, the left renal artery shows evidence of dissection without reentry in the course of the branch vessel. In this branch, there is formation of thrombus in the left renal artery false lumen, which in turn causes a static obstruction and resultant renal malperfusion. F, false lumen; SMA, superior mesenteric artery; T, true lumen.

onset (10). Data from the IRAD registry demonstrated a non-operative mortality rate of $24 \%$ by 24 hours, $29 \%$ by 48 hours, $44 \%$ by 7 days, and $49 \%$ by 14 days with medical management alone. If surgical intervention is pursued, the mortality rates decrease to $10 \%$ by 24 hours, $12 \%$ by 48 hours, $16 \%$ by 7 days, and $20 \%$ by 14 days $(10,11)$. In contrast, in-house mortality rate for type B dissections in one preliminary study was documented at $34 \%$ for individuals managed surgically, $11 \%$ if endovascular intervention is pursued, and $8.7 \%$ with medical management alone (12), although these numbers reflect differing medical risks at the time therapy is undertaken and uncertain reporting standards. Death is typically secondary to rupture, extension of dissection with coronary or great vessel involvement or visceral malperfusion, valvular dysfunction, or cardiac tamponade (13).

\section{Initial medical management}

Following initial evaluation and resuscitation, patients with aortic dissection should be promptly admitted to the intensive care unit; stringent blood pressure and heart rate control are critical to prevent extension and rupture. The ultimate objective is to reduce aortic wall sheer stress by regulating the patient's cardiac output (14). Beta-blockers are the principal medications utilized in initial medical management, with esmolol the most common intravenous agent utilized in the United States. Multiple synergistic medications may be necessary for adequate hemodynamic control (15). General guidelines stipulate a target systolic blood pressure of $100-120 \mathrm{mmHg}$ (except in patients presenting with paraplegia, where a systolic range of $120-130$ is generally employed) and a heart rate of $60-80$ beats per minute $(15,16)$. Nonetheless, this proposed paradigm is often dependent on pathophysiology of dissection and target blood pressure can vary if static or dynamic obstruction is present.

\section{Endovascular vs. surgical intervention}

Further management is dictated by the type and extent of dissection and the presence of complicating factors. Historically, Stanford type A dissections are treated surgically via sternotomy with the objective of reconstructing the aortic true lumen and preventing further expansion of the false lumen, often requiring aortic reconstruction with a prosthetic graft. Surgical intervention is extensive and may require coronary artery reimplantation and restoration of aortic valve competence with intra-operative utilization of hypothermic circulatory arrest and cardiopulmonary bypass (17). Adjunctive endovascular techniques have been employed more recently to treat type A dissection with introduction of branched endografts. Endovascular interventions have also been used for "retro-A dissections"; dissections with 
an entry tear distal to the left subclavian origin, in which the false lumen extends retrograde into the ascending aorta. The objective of taking this course would be to cover the primary dissection entry point to prevent further retrograde propagation or re-entry (14).

Uncomplicated type B aortic dissections can often be managed with medical therapy alone. Data from the INSTEAD (Investigation of Stent Grafts in Aortic Dissection) trial demonstrated no discrete difference in mortality between patients treated with medical therapy plus endovascular stent-graft placement and those treated with medical therapy at 1 -year $(18,19)$. Nonetheless, pre-emptive thoracic endovascular intervention [thoracic endovascular aortic repair (TEVAR)] is increasingly being used for uncomplicated dissection to avert late complications, such as aneurysmal false lumen degeneration and delayed rupture (20-22). Data from the more recently published 5-year follow-up INSTEAD XL study demonstrates a long-term benefit of TEVAR, with reduction in aorticspecific mortality and disease progression compared to medical therapy in a cohort of patients with uncomplicated dissections and non-emergent procedures (23).

For complicated type B aortic dissections, endovascular repair has become the principal modality of treatment, given the excess mortality of open repair (24). Placement of a stent-graft to cover the primary tear in anatomically suitable candidates maintains patency of the true lumen and in ideal cases causes depressurization and subsequent thrombosis of the false lumen. Previous indications for open surgical repair, including aneurysmal expansion, refractory pain, and malperfusion are now indications for endovascular intervention, given better expediency and safety (13). Mortality estimate for TEVAR recipients in cases of complicated dissection with end-organ malperfusion or rupture was $10.8 \%$ at 30 days and $29.4 \%$ at 1 year, similar to mortality rates for medically treated patients with uncomplicated dissection $(25,26)$.

\section{Endovascular management}

Interventionalists have multiple therapeutic endovascular options at their disposal for the treatment of aortic dissection, including dissection flap fenestration, stent graft placement, branch vessel stenting, or a combination of these techniques. This is predicated on the clinical presentation, dissection anatomy and the flow dynamics of the true and false lumens. The ultimate objective is to prevent aortic rupture and restore end-organ perfusion.
Adequate preoperative evaluation is critical. Preprocedural imaging often allows precise identification of the location of primary entry tears and secondary tears in the dissection flap, and provides critical information regarding aortic diameter, angulation and coverage length (facilitating the selection of appropriately sized stent grafts, stent graft limbs, and/or bare metal stents for endovascular repair). Relative to un-dissected landing zone, stent grafts should be oversized by $10-20 \%$ to ensure proper aortic wall apposition (27). Access vessel course, caliber, and degree of calcification should also be evaluated on preoperative imaging prior to sheath insertion.

False lumen patency contributes to aortic dilatation and is a predictor of late mortality (28). The intent of the stent graft is to prevent blood flow into the false lumen by deploying the device across the primary entry tear site. Blood is redirected into the true lumen through the graft, facilitating thrombosis and remodeling of the false lumen. This decreases the risk of subsequent aneurysmal degeneration and rupture (29).

\section{Dissection flap fenestration}

Percutaneous balloon fenestration functions to create a tear in the intimal flap, establishing local communication between the true and false lumens. If the true lumen remains collapsed, a stent is deployed in the true lumen to lift the flap off the opposite wall. This procedure increases true lumen perfusion and perfusion pressure while incidentally promoting flow in the false lumen. The principal role of dissection flap fenestration is to help restore perfusion to branches compromised by dynamic obstruction. In certain cases, it is performed to arrest the process of retrograde false lumen thrombosis, which, if untreated, can lead to devastating visceral and spinal malperfusion through occlusion of vessels originating in part or completely from the false lumen. Of note, this procedure is often employed at our institution to treat patients with acute type A dissection and prolonged malperfusion and patients at risk of reperfusion injury, prior to submitting patients to open repair with circulatory arrest (30).

The fenestration procedure is performed in conjunction with intravascular ultrasound (IVUS) and comparative manometry between the true lumen at the level of the aortic root and that of the true and false lumens at the level of the visceral segments and in the visceral and limb branches themselves. In concert with clinical and laboratory values, this technique provides the most definitive assessment of 


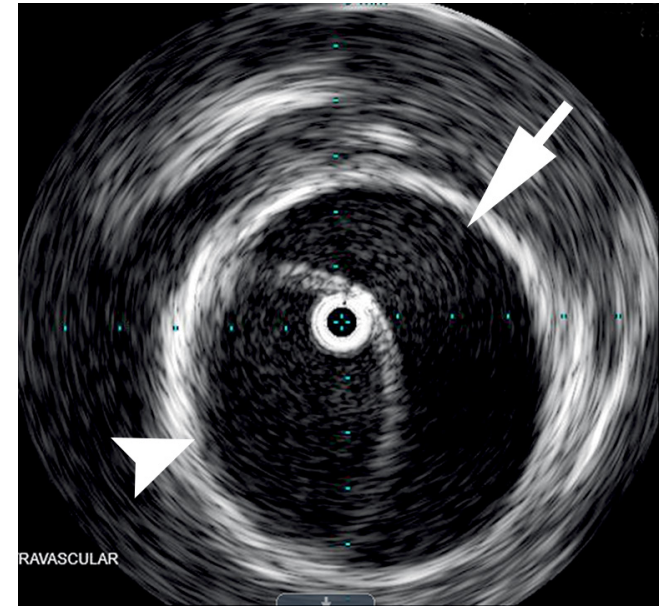

Figure 3 Intravascular ultrasound (IVUS) allows delineation of both the true (arrowhead) and false lumen (arrow). IVUS assessment for complete true lumen placement of the wires from each access site is critical for treatment of dissection. Additionally, true lumen thrombus may occasionally develop in these patients, which may be identified using IVUS and treated accordingly to prevent distal limb embolization when true lumen flow is restored.

ongoing arterial obstruction via visceral and limb perfusion pressures, and allows for subsequent stenting should malperfusion be identified. IVUS also allows identification of both false and true lumen thrombus, which are managed using differing techniques (Figure 3).

Scrutiny of the femoral access sites on pre-procedural CTA is important, allowing the interventionist to determine the presence or absence of access site dissection. A dissected common femoral artery is not a contraindication to access, but requires care in selecting and achieving true lumen access. Occasionally, obtaining true lumen access may require placement of a balloon over the aortic bifurcation into the target true lumen from the contralateral side and fluoroscopic puncture of the balloon, or a more distal initial access using the superficial femoral artery.

After gaining femoral access bilaterally, IVUS is employed to determine the location of the sheath and wire (true or false lumen). From one side, the wire is navigated under fluoroscopic and IVUS guidance to the aortic root true lumen, where a flush catheter is placed for reference manometry. A 5 French hydrophilic catheter (most commonly a Cobra 2 shape) is then used from the other access site to select the visceral branches for comparative pressure measurements. The superior mesenteric artery
(SMA) is considered the most critical and is assessed first (given the high morbidity and mortality rates associated with bowel malperfusion), followed by the renal arteries. The celiac artery may be assessed if a dissection of the ostium is present, but in our clinical experience, celiac flow compromise is often clinically well-tolerated if SMA perfusion is adequate. Small contrast injections (1-2 $\mathrm{mL} /$ second hand injection rate for a total contrast volume of 3-4 $\mathrm{mL}$ ) are utilized to assess whether the catheter has been placed beyond any branch vessel dissection or re-entry tear, providing a true assessment of end-organ perfusion pressure. Smaller caliber IVUS (5 or $6 \mathrm{~F})$ can be employed for anatomic delineation of branch vessel dissection as necessary. Generally, a $15 \mathrm{mmHg}$ systolic (or $10 \mathrm{~mm}$ mean) pressure gradient between the root and the branch is considered significant, particularly if there are associated clinical signs of malperfusion. However, this threshold is not rigid, and in the setting of marginal perfusion deficits, the decision to intervene depends on dissection type and whether aortic root surgery is imminent, the particular vessel involved, and the patient's propensity to adhere to antihypertensive therapy.

Should dynamic visceral malperfusion be identified, aortic fenestration is then performed. This involves creation of a hole through the dissection flap at or just above the celiac ostium using a long needle such as a Rosch-Uchida needle (Cook Medical, Bloomington, Indiana, USA). The direction of the needle puncture is determined using real time IVUS guidance at the level of the needle tip, which is directed through the dissection flap, generally from the smaller true to the larger false lumen (31). After removing the stylet, a guidewire is then advanced from true to false lumen, which is again confirmed with IVUS. A $16 \mathrm{~mm}$ angioplasty balloon is centered across the flap and inflated to widen the fenestration and promote flow from false-totrue lumen (Figure 4) (32). If true lumen collapse persists, a 16 or $18 \mathrm{~mm}$ self-expanding stent is then deployed exclusively in the true lumen above the fenestration after pulling the wire back into the true lumen and advancing above the tear (Figures 5,6). A potential limitation of dissection flap fenestration is persistent flow into the false lumen, but vulnerability to future aneurysmal degeneration and risk of rupture is considered an acceptable trade-off in the setting of malperfusion (14).

Limb ischemia as a complication of aortic dissection most commonly occurs through one of two mechanisms: the abdominal aortic dissection flap covering the common iliac artery true lumen, or iliac artery dissection without 


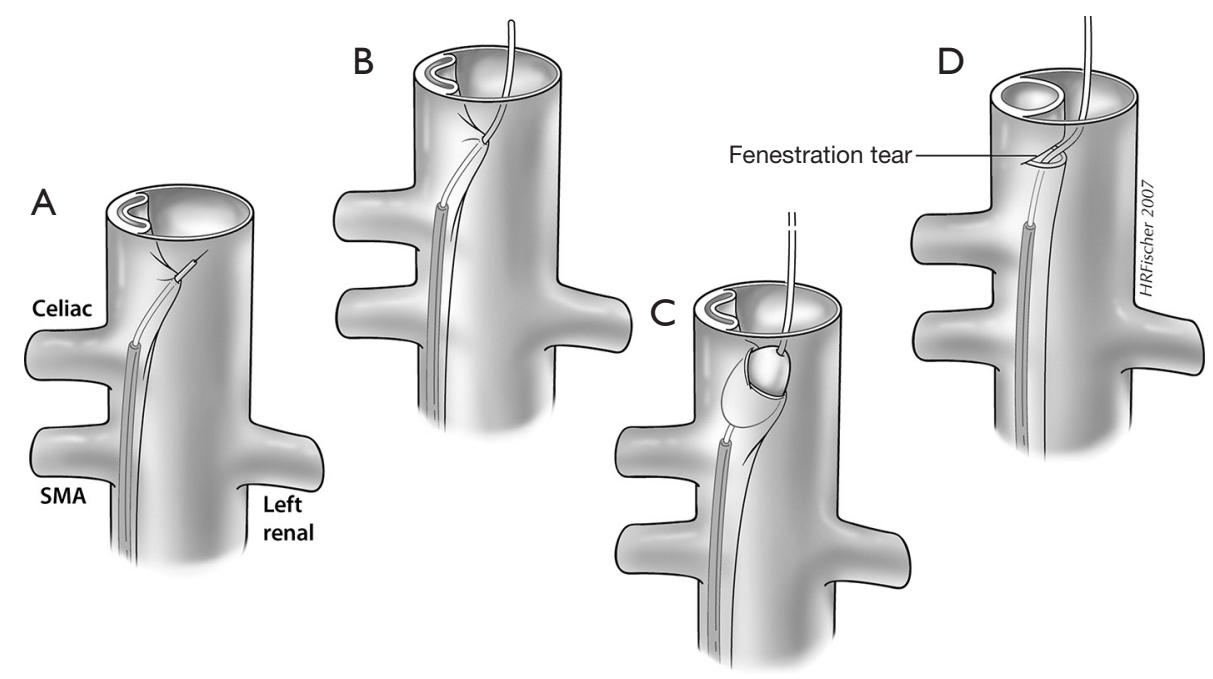

Figure 4 Image depiction of a Rosch-Uchida needle used to traverse the dissection flap from the true to the false lumen with IVUS. The direction of the needle puncture is determined using real time IVUS guidance at the level of the needle tip, which is directed through the dissection flap, generally from the smaller true to the larger false lumen (A). After advancing the catheter over the stylet into the false lumen, the stylet is removed and a wire is placed into the false lumen (B). A $16 \mathrm{~mm}$ angioplasty balloon is centered across the flap and inflated to widen the fenestration and promote flow from false-to-true lumen $(\mathrm{C})$, thus creating a fenestration (D). IVUS, intravascular ultrasound; SMA, superior mesenteric artery.

re-entry leading to thrombosed false lumen (which fills the vessel cross-section and obliterates the true lumen). Treatment generally requires infra-renal aortic fenestration with aortoiliac stenting. IVUS assessment for complete true lumen placement of the wires from each access site is critical for treatment of dissection-related limb ischemia using these techniques. Additionally, true lumen thrombus may occasionally develop in these patients, which must be identified using angiography and IVUS and treated accordingly to prevent distal limb embolization when true lumen flow is restored. At our institution, the most common method of treatment for true lumen thrombosis involves the use of rheolytic thrombectomy with the AngioJet device (Boston Scientific-Marlborough, MA, USA). Vascular surgery consultation is suggested for all patients with prolonged limb malperfusion either before or after this procedure, as reperfusion injury and compartment syndrome may develop, possibly requiring fasciotomy or additional adjunctive procedures.

\section{TEVAR}

Given the lack of widespread operator expertise in fenestration techniques, as well as the time-consuming and complex nature of this procedure, endograft therapy has increased in popularity as a procedural alternative. The therapeutic benefit through endograft deployment is achieved by coverage of the primary entry tear, promoting flow in the true lumen in hopes of achieving false lumen decompression and restoration of downstream flow. Hemodynamic studies indicate that obliteration of the false lumen by stentgraft deployment may be more effective in improving malperfusion states in ex vivo dissection models compared to fenestration (33). This is supported by the IRAD database, as malperfusion states improved in 9 of 18 patients treated with endovascular fenestration compared to 16 of 17 patients treated with endograft therapy (12). This evidence is limited by an extremely small number of patients, however, and fenestration should still be in the armamentarium of every center treating the full spectrum of aortic disease. This is particularly true in patients without an identifiable entry tear, inappropriate anatomy for stent grafting, or in patients with multiple tears or complex flap anatomy.

Multiple stent-grafts are available on the market for endovascular aortic repair. All thoracic stent-grafts are selfexpanding, with the skeleton consisting of metallic Nitinol (Gore-Flagstaff, AZ, USA; Medtronic-Minneapolis, MN, USA; Bolton-Sunrise, FL, USA) or stainless steel (CookBloomington, IN, USA) with an external membrane made of polytetrafluoroethylene (Gore) or polyester (Medtronic, 


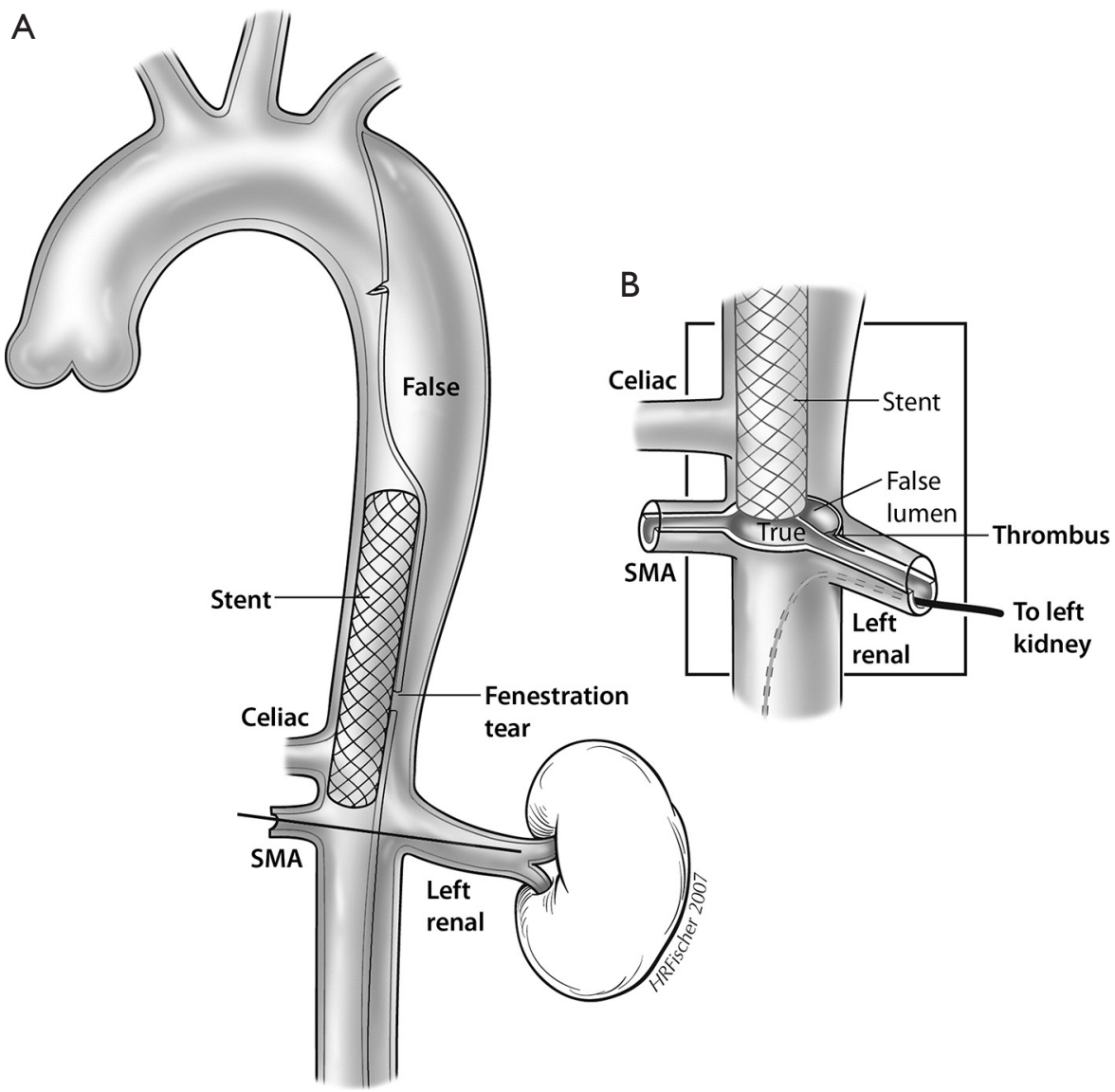

Figure 5 Although fenestration can equalize pressures across the dissection flap, self-expanding 16- to 18-mm-diameter Wallstents (Boston Scientific-Marlborough, MA) are usually placed to buttress open the aortic true lumen and are typically placed near the compromised branch vessel (A). These are usually inserted via the already obtained percutaneous access sheaths and deployed by IVUS guidance. Care is taken to withdraw the guidewire from across the fenestration tear and re-advance it within the aortic true lumen, so as to deploy the stents exclusively within the aortic true lumen, rather than straddle the tear from true to false lumen. Fenestration and stenting of the aortic true lumen treats the dynamic obstruction of the depicted mesenteric vessels, but not the static obstruction seen in the depicted left renal artery. In this branch, pressure measurements confirm a significant $(>20 \mathrm{mmHg})$ systolic gradient requiring additional treatment (B). SMA, superior mesenteric artery.

Cook, Bolton). The proximal and distal ends of the stentgrafts can be bare or covered, depending on the vendor.

According to the instructions for use, the deployed stent-graft should extend 10-20 mm proximal and distal to the site of intimal tear and should land in relatively nondiseased aortic segments to obtain an adequate seal. Wall apposition is critical to prevent endoleak, persistent false lumen perfusion or device migration. Unfortunately, these requirements may necessitate coverage of one or more of the arch branch vessels. If this is anticipated preoperatively, then a carotid and vertebral artery duplex ultrasound or CTA can be performed to assess the anatomy and flow relationships of the cerebrovascular vasculature. If stent deployment is anticipated to jeopardize left subclavian perfusion, treatment would require use of a single-side branch stent graft, carotid-subclavian bypass, subclavian transposition, or coverage of the subclavian origin with postoperative bypass, if needed (34). Preemptive embolization of the native subclavian artery may be necessary to prevent endoleak (35-37). Endovascularly fenestrated or branched stent graft techniques have been devised to establish flow to great vessels that require coverage by the endograft, but these are not yet widely available (38-40). In the setting of malperfusion, it is sometimes more expeditious to perform a fenestration, since dynamic obstruction of the SMA can be diagnosed and corrected within 1 hour. 

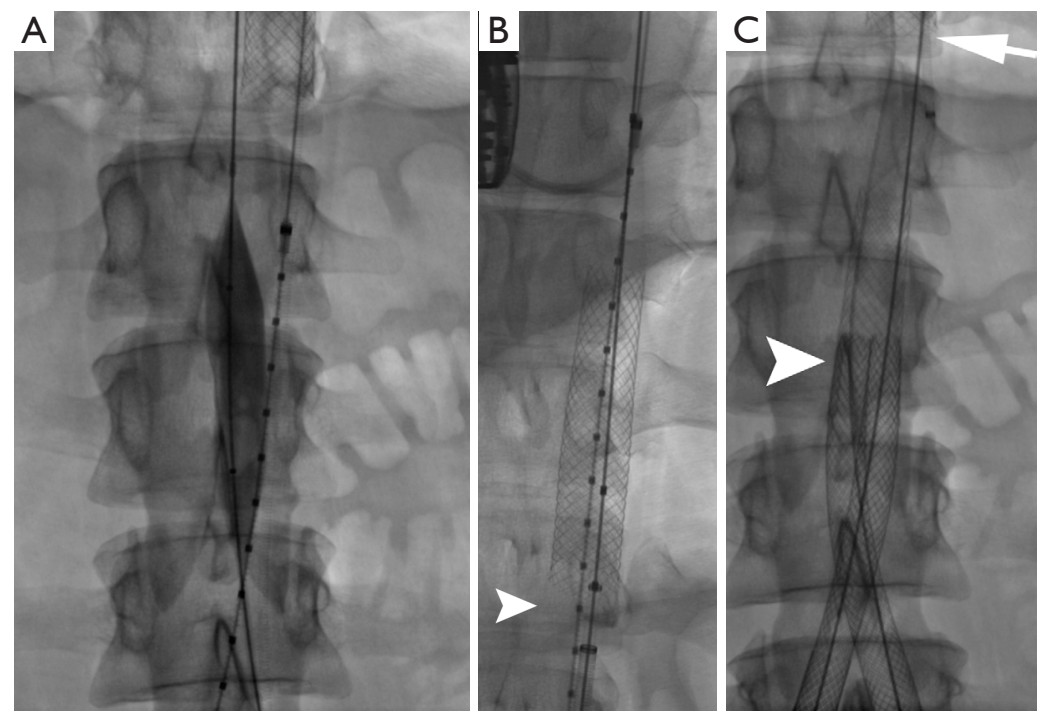

Figure 6 Intra-procedural fluoroscopy images of a type B aortic dissection. (A) An IVUS catheter can be seen in the true lumen, which helped ascertain directionality of the needle puncture during fenestration. An angioplasty balloon is centered across the flap and inflated to widen the fenestration and promote flow from false-to-true lumen. A subtle waist can be identified at the site of puncture. (B) A supra-celiac Wallstent is deployed to maintain patency of the previously collapsed true lumen. Pre-deployment IVUS can help with stent positioning. (C) The caudal aspect of the supra-celiac true stent can be seen at the top of the image (arrow). This patient also underwent a second infrarenal fenestration (not shown), and is now being treated with true lumen aortoiliac stenting (arrowhead). IVUS, intravascular ultrasound.

Another preoperative consideration is placement of a lumbar drain if extensive coverage of the thoracic aorta is expected or if the patient has undergone previous abdominal aortic surgery or endografting. By decreasing the cerebrospinal fluid (CSF) pressure, blood flow toward the spinal cord is augmented, decreasing the risk of paraplegia if the artery of Adamkiewicz or its collaterals are covered by the stent graft $(41,42)$.

TEVAR is typically performed under general anesthesia, providing for respiratory control and more detailed imaging. If femoral vasculature is confirmed to be patent and adequate in caliber, the procedure is performed percutaneously via preclose technique (43) or by groin cut down. If tortuosity or diminutive luminal caliber is a concern, iliac artery pre-rupture with covered stent grafting or use of a retroperitoneal conduit can be considered. Nonetheless, in our experience, patients with dissections typically have ecstatic iliac arteries and diameter of the access artery is rarely a treatment impediment. Ultimately, a stiff wire is advanced into the true lumen of the proximal thoracic aorta. IVUS can be introduced to confirm exclusive true lumen wire location. The fluoroscopic detector is positioned in the left anterior oblique position to better profile the aortic arch and landing zones, which are delineated angiographically (and with IVUS in our institution). The device delivery system is advanced over the wire and properly positioned (Figure 7). Ventilatory suspension by anesthesia and medical control of blood pressure can be pursued to optimize precise deployment $(26,44)$. Post-deployment angiography is performed to evaluate for endoleak. Balloon dilatation to help ensure stent apposition to the aortic wall is not advisable in cases of acute dissection, as this can create fenestrations or increase risk of extension of the dissection or aortic rupture. The sheath and deployment device are thereupon removed and the arteriotomy is closed.

\section{Branched and non-branched endovascular repair}

The aortic arch and subdiaphragmatic aorta is complex due to the plurality of critical branch configurations. This anatomic complexity is exacerbated in endovascular aortic repair, particularly if the disease-free segment of the aorta is short. To circumvent this problem, endovascular fenestrated and branched stent-graft techniques have been devised to help with vessel incorporation in complex cases.

Branched endografts are custom-made devices with side-branch components designed to precisely fit into the 

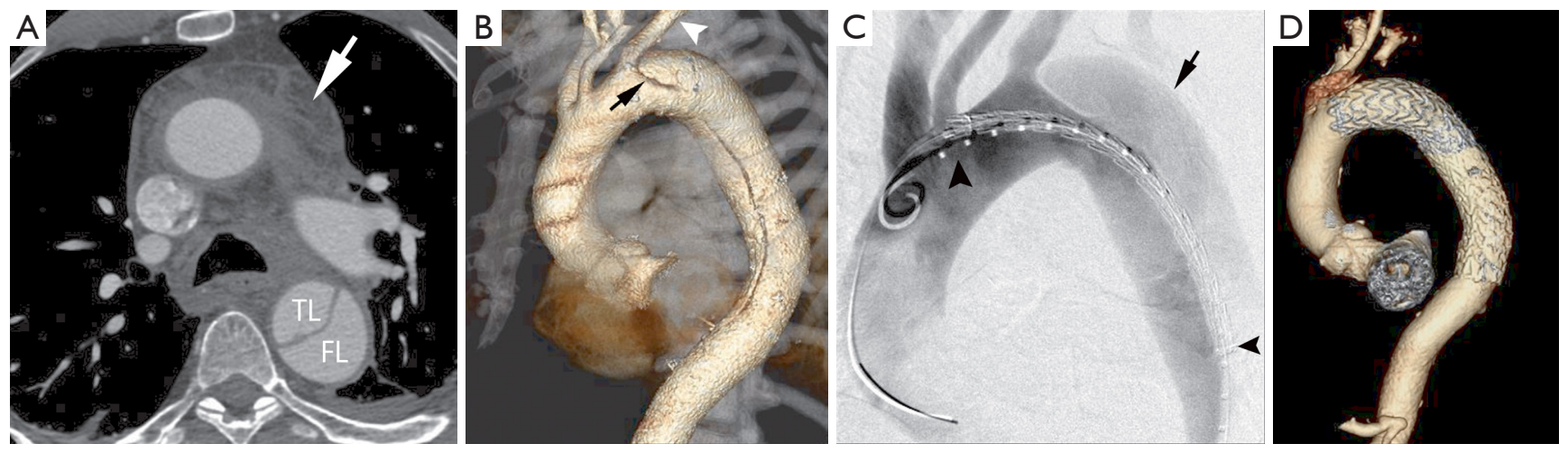

Figure 7 A 67-year-old man presents with acute onset of sharp chest pain radiating to back. (A) Contrast enhanced CT angiography (CTA) confirms an aneurysmal type B aortic dissection with true lumen (TL) and false lumen (FL) delineated. There is evidence of acute aortic rupture with mediastinal hematoma (arrow). (B) 3D CTA reconstruction demarcating the extent of aortic dissection lateral to the left subclavian artery origin (black arrow) with extension to the supra-celiac descending thoracic aorta. The left subclavian artery is denoted by the white arrowhead. (C) Intra-procedural fluoroscopy images demonstrating an Omni Flush catheter with radiopaque markers and an aortic stent graft (black arrowheads), not yet deployed. The device delivery system is advanced over a stiff wire and properly positioned. The fluoroscopic detector is positioned in the left anterior oblique position to better profile the aortic arch and landing zones, which are delineated angiographically and with IVUS at our institution. The black arrow denotes the false lumen. (D) Post-TEVAR 3D CTA reconstruction demonstrates a deployed aortic stent graft maintaining patency of the true lumen. TEVAR, thoracic endovascular aortic repair; IVUS, intravascular ultrasound.

necessary aortic branch vessels, with appropriate branch diameter and length to facilitate branch vessel seal. This underscores the importance of preoperative imaging to evaluate branch vessel anatomy. Multiple manufacturers currently produce investigational branched endograft devices, including Gore Medical (Flagstaff, AZ, USA), Cook Medical (Bloomington, IN, USA), Bolton Medical (Sunrise, FL, USA), and Medtronic Vascular (Minneapolis, MN, USA). The branch components may be covered or uncovered and self-expanding or balloon-expandable. Using combined femoral and brachial or axillary approach, pre-loaded catheters and guide-wires are utilized for implantation of the branched endografts at the origins of the target vessel, with subsequent placement of covered stents from the branches into the more distal segments of the grafted vessels. Branched endografts remain investigational in the United States and their role is perhaps more pertinent in endovascular repair of type B dissections.

\section{Conclusions}

The treatment paradigm for aortic dissection is complex and dependent on acuity of presentation and clinical and anatomic considerations. Certainly, anatomic location previously stratified patients into surgical and medical management candidates, but the advent of endovascular techniques continues to transform this paradigm. Initial blood pressure and heart rate control is critical to prevent propagation of dissection. Type A dissections usually require surgical intervention, but patients may benefit from preoperative fenestration. Uncomplicated type B aortic dissections can often be managed with medical therapy, but many exceptions to this exist. In contrast, endovascular repair has become the principle modality of treatment for complicated type B aortic dissections. This includes stent-graft placement, dissection flap fenestration, branch vessel stenting, or a combination of these techniques. The ultimate objective is to prevent aortic rupture and restore end-organ perfusion. Though morbidity and mortality for endovascular procedures is comparatively lower compared to more extensive surgical options, continued enhancement of endovascular techniques and devices is needed $(24,45)$.

\section{Acknowledgements}

None.

\section{Footnote}

Conflicts of Interest: The authors have no conflicts of interest to declare. 


\section{References}

1. Vilacosta I, Aragoncillo P, Canadas V, et al. Acute aortic syndrome: a new look at an old conundrum. Heart 2009;95:1130-9.

2. Baguet JP, Chavanon O, Sessa C, et al. European Society of Hypertension scientific newsletter: hypertension and aortic diseases. J Hypertens 2012;30:440-3.

3. Cregler LL. Aortic dissection and cocaine use. Am Heart J 1992;124:1665.

4. Attias D, Stheneur C, Roy C, et al. Comparison of clinical presentations and outcomes between patients with TGFBR2 and FBN1 mutations in Marfan syndrome and related disorders. Circulation 2009;120:2541-9.

5. Olsson C, Thelin S, Ståhle E, et al. Thoracic aortic aneurysm and dissection: increasing prevalence and improved outcomes reported in a nationwide populationbased study of more than 14,000 cases from 1987 to 2002 . Circulation 2006;114:2611-8.

6. Acosta S, Ogren M, Bengtsson H, et al. Increasing incidence of ruptured abdominal aortic aneurysm: a population-based study. J Vasc Surg 2006;44:237-43.

7. Mehta RH, Manfredini R, Hassan F, et al. Chronobiological patterns of acute aortic dissection. Circulation 2002;106:1110-5.

8. Cigarroa JE, Isselbacher EM, DeSanctis RW, et al. Diagnostic imaging in the evaluation of suspected aortic dissection. Old standards and new directions. N Engl J Med 1993;328:35-43.

9. Williams DM, Lee DY, Hamilton BH, et al. The dissected aorta: part III. Anatomy and radiologic diagnosis of branch- vessel compromise. Radiology 1997;203:37-44.

10. Hagan PG, Nienaber CA, Isselbacher EM, et al. The International Registry of Acute Aortic Dissection (IRAD): new insights into an old disease. JAMA 2000;283:897-903.

11. Mehta RH, Suzuki T, Hagan PG, et al. Predicting death in patients with acute type a aortic dissection. Circulation 2002;105:200-6.

12. Fattori R, Tsai TT, Myrmel T, et al. Complicated acute type $\mathrm{B}$ dissection: is surgery still the best option? A report from the International Registry of Acute Aortic Dissection (IRAD). JACC Cardiovasc Interv 2008;1:395-402.

13. Nienaber CA, Clough RE. Management of acute aortic dissection. Lancet 2015;385:800-11.

14. Patel PJ, Grande W, Heib RA. Endovascular management of acute aortic syndromes. Seminars in Interventional Radiology 2011:28:10-23.

15. Suzuki T, Isselbacher EM, Nienaber CA, et al. Type- selective benefits of medications in treatment of acute aortic dissection (from the International Registry of Acute Aortic Dissection [IRAD]). Am J Cardiol 2012;109:122-7.

16. Lu Q, Feng J, Zhou J, et al. Endovascular repair of ascending aortic dissection: a novel treatment option for patients judged unfit for direct surgical repair. J Am Coll Cardiol 2013;61:1917-24.

17. Chiu P, Miller DC. Evolution of surgical therapy for Stanford acute type A aortic dissection. Ann Cardiothorac Surg 2016;5:275-95.

18. Nienaber CA, Rousseau H, Eggebrecht H, et al. Randomized comparison of strategies for type B aortic dissection: the INvestigation of STEnt Grafts in Aortic Dissection (INSTEAD) trial. Circulation 2009; 120:2519-28.

19. Ulug P, McCaslin JE, Stansby G, et al. Endovascular versus conventional medical treatment for uncomplicated chronic type B aortic dissection. Cochrane Database Syst Rev 2012;11:CD006512.

20. Patterson B, Holt P, Nienaber C, et al. Aortic pathology determines midterm outcome after endovascular repair of the thoracic aorta: report from the Medtronic Thoracic Endovascular Registry (MOTHER) database. Circulation 2013;127:24-32.

21. Fattori R, Montgomery D, Lovato L, et al. Survival after endovascular therapy in patients with type B aortic dissection: a report from the International Registry of Acute Aortic Dissection (IRAD). JACC Cardiovasc Interv 2013;6:876-82.

22. Jia X, Guo W, Li TX, et al. The results of stent graft versus medication therapy for chronic type B dissection. J Vasc Surg 2013;57:406-14.

23. Nienaber CA, Kische S, Rousseau H, et al. Endovascular repair of type $\mathrm{B}$ aortic dissection: long-term results of the randomized investigation of stent grafts in aortic dissection trial. Circ Cardiovasc Interv 2013;6:407-16.

24. Trimarchi S, Nienaber CA, Rampoldi V, et al. Role and results of surgery in acute type $B$ aortic dissection: insights from the International Registry of Acute Aortic Dissection (IRAD). Circulation 2006;114:I357-64.

25. White RA, Miller DC, Criado FJ, et al. Report on the results of thoracic endovascular aortic repair for acute, complicated, type $\mathrm{B}$ aortic dissection at 30 days and 1 year from a multidisciplinary subcommittee of the Society for Vascular Surgery Outcomes Committee. J Vasc Surg 2011;53:1082-90.

26. Patel AY, Eagle KA, Vaishnava P. Acute type B aortic dissection: insights from the International Registry of Acute Aortic Dissection. Ann Cardiothorac Surg 
2014;3:368-74.

27. Rousseau H, Cosin O, et al. Endovascular treatment of thoracic dissection. Semin Intervent Radiol 2007;24:167-79.

28. Bernard Y, Zimmerman H, Chocron S, et al. False lumen patency as a predictor of late outcome in aortic dissection. Am J Cardiol 2001;87:1378-82.

29. DiMusto PD, Williams DM, Patel HJ, et al. Endovascular management of type B aortic dissections. J Vasc Surg 2010;52:26S-36S.

30. Yang B, Patel HJ, Williams DM, et al. Management of type A dissection with malperfusion. Ann Cardiothorac Surg 2016;5:265-74.

31. Williams DM, Lee DT, Hamilton BH, et al. The dissected aorta: percutaneous treatment of ischemic complications-principles and results. J Vasc Interv Radiol 1997;8:605-25.

32. Slonim SM, Miller DC, Mitchell RS, et al. Percutaneous balloon fenestration and stenting for life-threatening ischemic complications in patients with acute aortic dissection. J Thorac Cardiovasc Surg 1999;117:1118-26.

33. Vedantham S, Picus D, Sanchez LA, et al. Percutaneous management of ischemic complications in patients with type-B aortic dissection. J Vasc Interv Radiol 2003;14:181-94.

34. Harris PL, Buth J. An update on the important findings from the EUROSTAR EVAR registry. Vascular 2004;12:33-8.

35. Nation DA, Wang GJ. TEVAR: endovascular repair of the thoracic aorta. Semin Intervent Radiol 2015;32:265-71.

36. Zhou $W$, Reardon $M$, Peden EK, et al. Hybrid approach to complex thoracic aortic aneurysms in high-risk patients: surgical challenges and clinical outcomes. J Vasc Surg

Cite this article as: Khayat M, Cooper KJ, Khaja MS, Gandhi R, Bryce YC, Williams DM. Endovascular management of acute aortic dissection. Cardiovasc Diagn Ther 2018;8(Suppl 1):S97-S107. doi: 10.21037/cdt.2017.10.07
2006;44:688-93.

37. Bergeron P, Mangialardi N, Costa P, et al. Great vessel management for endovascular exclusion of aortic arch aneurysms and dissections. Eur J Vasc Endovasc Surg 2006;32:38-45.

38. Shahverdyan R, Gawenda M, Brunkwall J. Triple-barrel graft as a novel strategy to preserve supra-aortic branches in arch-TEVAR procedures: clinical study and systematic review. Eur J Vasc Endovasc Surg 2013;45:28-35.

39. Tolenaar JL, van Keulen JW, Trimarchi S, et al. The chimney graft, a systemic review. Ann Vasc Surg 2012;26:1030-8.

40. Anthony Lee W. Status of branched grafts for thoracic aortic arch endovascular repair. Semin Vasc Surg 2016;29:84-9.

41. Cheung AT, Weiss SJ, McGarvey ML, et al. Interventions for reversing delayed-onset postoperative paraplegia after thoracic aortic reconstruction. Ann Thorac Surg 2002;74:413-9; discussion 420-1.

42. Khoynezhad A, Donayre CE, Bui H, et al. Risk factors of neurologic deficit after thoracic aortic endografting. Ann Thorac Surg 2007;83:S882-9; discussion S890-2.

43. Lee WA, Brown MP, Nelson PR, et al. Total percutaneous access for endovascular aortic aneurysm repair ("Preclose" technique). J Vasc Surg 2007;45:1095-101.

44. Nienaber CA, Kische S, Rehders TC, et al. Rapid pacing for better placing: comparison of techniques for precise deployment of endografts in the thoracic aorta. J Endovasc Ther 2007;14:506-12.

45. Parker JD, Golledge J. Outcome of endovascular treatment of acute type B aortic dissection. Ann Thorac Surg 2008;86:1707-12. 